\title{
Aggravation of left ventricular diastolic dysfunction in hypertensives with coronary artery disease
}

This article has been corrected since Advance Online Publication, and a corrigendum is also printed in this issue.

\author{
Ioannis Vlasseros ${ }^{1}$, Vasiliki Katsi ${ }^{1}$, Gregory Vyssoulis ${ }^{2}$, Ioannis Pylarinos ${ }^{1}$, Dimitrios Richter ${ }^{3}$, \\ Theodoros Gialernios ${ }^{1}$, Georgios Souretis ${ }^{1}$, Dimitris Tousoulis ${ }^{2}$, Christodoulos Stefanadis ${ }^{2}$ and \\ Ioannis Kallikazaros ${ }^{1}$
}

We investigated the combined effects of hypertension and coronary artery disease (CAD) on left ventricular (LV) diastolic function. We examined 118 consecutive hypertensives who underwent diagnostic coronary angiography. All patients underwent a complete echocardiographic study within $24 \mathrm{~h}$ of catheterization by operators blind to their condition. The study participants were divided into two groups according to the presence of CAD: group A, with the disease $(n=72)$; and group B, without $(n=46)$. Patients with CAD exhibited lower LV fractional shortening and ejection fraction $(P=0.002$ and $P=0.001)$. Hypertensives with CAD had significantly prolonged isovolumic relaxation time (IVRT) compared to those without CAD $(P<0.001)$. Most interestingly, CAD patients had significantly worse Ema/Ama, Vp (flow propagation velocity), E/Np and Vp/ IVRT (all $\boldsymbol{P}<0.05$ ). In addition, after adjusting for confounders, univariate and multivariate logistic regression analyses revealed that IVRT increases were associated with greater odds of CAD, whereas decreases in Vp or Vp/IVRT were associated with lower odds of CAD (all $P \leqslant 0.001$ ). In hypertensives, the early recognition of LV diastolic performance alteration may be associated with the presence of significant CAD, indicating the need for more aggressive approaches both in terms of pharmacological treatment and interventional evaluation.

Hypertension Research (2013) 36, 885-888; doi:10.1038/hr.2013.67; published online 11 July 2013

Keywords: coronary artery disease; diastolic dysfunction; echocardiographic indices

\section{INTRODUCTION}

Left ventricular (LV) diastolic dysfunction represents a common pathophysiologic consequence of arterial hypertension that can be detected in the early stages of the disease, even before the development of LV hypertrophy. ${ }^{1}$ In addition to hypertension, myocardial ischemia is commonly observed with LV diastolic dysfunction, primarily the delayed relaxation pattern. ${ }^{2}$

Derangement of LV diastolic properties in hypertensive subjects has been linked to adverse cardiovascular outcomes including the augmented risk of heart failure with preserved ejection fraction ${ }^{3,4}$ and atrial fibrillation. ${ }^{5}$ Moreover, some authors have called for more aggressive treatment of hypertensives with impaired LV diastolic function in terms of improved prognosis. ${ }^{6}$

The estimation of LV diastolic properties in daily clinical practice is performed with the use of established echocardiographic indices that are primarily derived from mitral inflow Doppler studies. However, because these are dependent on LV loading conditions, other techniques such as tissue Doppler imaging have gained interest due to their more sensitive detection of $\mathrm{LV}$ diastolic dysfunction in hypertensive patients. ${ }^{7,8}$
The non-invasive detection of coronary artery disease (CAD) remains an important goal in the management of hypertensive subjects. The aim of the present study was to investigate the plausible additional role of echocardiographic LV diastolic dysfunction indices in the determination of hypertensives with CAD.

\section{METHODS}

Study population

We examined 136 consecutive hypertensive patients from the outpatient hypertensive clinic of our institution who underwent coronary artery angiography to evaluate chest pain.

Patients with a known history of myocardial infarction as well as those with congenital heart disease, clinically significant valvular disease, cardiomyopathy, chronic obstructive pulmonary disease, renal insufficiency (serum creatinine $>1.5 \mathrm{mg} \mathrm{dl}^{-1}$ ), AV conduction disturbances, atrial fibrillation and diabetes mellitus were excluded from the present study.

Thus, the final study cohort comprised 118 hypertensive subjects (mean age $63 \pm 8$ years, 77 male) who, according to the study protocol, underwent a complete echocardiographic examination with special focus on the estimation

${ }^{1}$ Cardiology Department, Hippokration Hospital of Athens, Athens, Greece; ${ }^{2} 1$ st Cardiology Unit, Athens University Medical School, Hippokration Hospital, Athens, Greece and ${ }^{3}$ Cardiology Department, Euroclinic Hospital of Athens, Athens, Greece

Correspondence: Dr V Katsi, Cardiology Department, Hippokration Hospital of Athens, Lefkados 10, 11362 Athens, Greece.

E-mail: vkkatsi@yahoo.gr

Received 23 October 2012; revised 17 January 2013; accepted 18 March 2013; published online 11 July 2013 
of LV diastolic function, with the examiners blinded to the catheterization results. The study protocol was approved by the ethics committee of our institution and complied with the Declaration of Helsinki. All participants provided written informed consent.

\section{Office BP measurement}

Sitting BP was measured three times in the right arm of the individual with a mercury sphygmomanometer after a 5-min rest and with at least a 1-min interval between readings. The mean value of the last two measurements was accepted, discarding the first. Hypertension was defined as systolic BP $\geqslant 140 \mathrm{~mm} \mathrm{Hg}$, diastolic BP $\geqslant 90 \mathrm{~mm} \mathrm{Hg}$ or the use of antihypertensive medication. Patients with secondary hypertension were excluded from the study

\section{Anthropometric and biochemical parameters}

Body weight and height were measured according to established methods, and body mass index and body surface area were calculated according to standard formulae. In addition, all participants underwent venous blood sampling for the determination of the typical biochemical parameters, including the lipidemic profile, serum glucose and creatinine, after an overnight fast.

\section{Echocardiographic study}

Echocardiograms were performed by two cardiac ultrasonographers, who had $>5$ years of experience with the Vivid 7 equipment (GE, Vigmed Ultrasound, Horten, Norway), using an adult matrix probe under continuous electrocardiographic guidance. Data analysis was performed offline by two independent expert level III blinded observers.

A two-dimensionally guided M-mode LV study was performed along the left parasternal long axis and LV end-systolic and end-diastolic diameter. LV posterior wall and intrarventricular septal thickness measurements were performed according to the guidelines of the American Society of Echocardiography ${ }^{9}$ in five consecutive cardiac cycles, obtaining the mean value of each parameter.

LV mass was estimated according to the formula of Deveraux and Reichek ${ }^{10}$ and was divided by body surface area for the calculation of the LV mass index. Moreover, we measured the mitral inflow maximal velocities of the $\mathrm{E}$ and $\mathrm{A}$ waves, as well as the isovolumic relaxation time (IVRT) and E wave deceleration time (DT), based on established methodology. ${ }^{9}$ Each of the aforementioned parameters was estimated in five consecutive cardiac cycles, and the mean value was obtained for the purposes of our study.

Color M-mode was used for the estimation of transmitral flow propagation velocity, which is an index of relaxation independent of the LV loading conditions.

Tissue Doppler imaging was performed by placing the sample volume in the endocardial surface of the basal lateral LV segment in the apical four-chamber echocardiographic view. Maximal Em and Am velocities were measured in five consecutive cardiac cycles, and the mean value was obtained for the purpose of the present study.

\section{Coronary artery angiography}

Coronary artery angiography was performed by the femoral route, using the standard JL4 and JR4 diagnostic catheters in nearly all cases. A coronary lesion was defined as hemodynamically significant if the normal coronary vessel lumen diameter was narrowed by $\geqslant 60 \%$.

\section{Statistical analysis}

Categorical variables are expressed as percentages, and continuous variables are expressed as the mean \pm standard deviation if they are normally distributed or as medians (range) in the case of non-normal distributions. We used the $t$-test for independent samples to compare the means of continuous variables or the Mann-Whitney $U$-test for non-normally distributed variables and the $\chi^{2}$-test for qualitative variables. Continuous variables were tested for normal distribution using the Shapiro-Wilk test for normality. Logistic regression was used to evaluate the association between echocardiographic indices on the likelihood of having CAD. The results are expressed in crude odds ratios and odds ratios adjusted for several confounders with corresponding $95 \%$ confidence intervals. Deviance residuals and the Hosmer-Lemeshow criterion were used to evaluate the goodness of fit of the logistic models.

Receiver operating characteristic curves of the IVRT, Vp, Em and Vp/IVRT and the corresponding areas under the curve were used for the discriminative ability of each echocardiographic index between CAD and non-CAD group patients. All findings were considered statistically significant at $P<0.05$.

\section{RESULTS}

The participants in this study were divided into two groups according to the presence of $\mathrm{CAD}$, based on the results of diagnostic coronary angiography: group $\mathrm{A}$, with the disease $(n=72)$; and group $\mathrm{B}$, without $(n=46)$

The two groups did not differ in the usual demographic and clinical parameters except for gender (Table 1). Male gender was more frequent in group A than in group B (75\% vs $50 \%$, respectively) ( $P=0.005)$. Moreover, no significant difference was recorded between the two groups in terms of the received antihypertensive regimens $(P>0.05)$.

The echocardiographic structural indices in the two groups are reported in Table 2. No differences were found in LV interventricular septal thickness, LV posterior wall thickness, LV mass index or relative wall thickness between the two groups (all $P>0.05$ ). However, patients with $\mathrm{CAD}$ exhibited lower LV fractional shortening and ejection fraction $(P=0.002$ and $P=0.001$, respectively), although they remained within the range of preserved LV systolic function (Table 2). Hypertensives with CAD had significantly prolonged IVRT compared to those without CAD $(129.2 \pm 13.2$ vs $110.5 \pm 14.5 \mathrm{~ms}$, $P<0.001)$

Table 1 Patient characteristics of the two study groups

\begin{tabular}{lccc}
\hline & Group A $(\mathrm{n}=72)$ & Group B $(\mathrm{n}=46)$ & P-value \\
\hline Age (years) & $63.6 \pm 9.5$ & $61.6 \pm 8.2$ & 0.224 \\
Male sex (\%) & $54(75 \%)$ & $23(50 \%)$ & $0.005^{*}$ \\
Smoking status (\%) & $37(51.4 \%)$ & $20(43.5 \%)$ & 0.402 \\
Dyslipidemia (\%) & $33(45.8 \%)$ & $23(50 \%)$ & 0.658 \\
BMI (kg m ${ }^{-2}$ ) & $27.9 \pm 4.1$ & $28.4 \pm 3.9$ & 0.521 \\
SBP (mm Hg) & $141 \pm 13$ & $138 \pm 7$ & 0.129 \\
DBP (mm Hg) & $82 \pm 8$ & $80 \pm 8$ & 0.259 \\
Pulse pressure (mm Hg) & $59 \pm 12$ & $58 \pm 8$ & 0.479 \\
Hypertension history (months) & $48(441)$ & $60(239)$ & 0.532
\end{tabular}

Abbreviations: BMI, body mass index; DBP, diastolic blood pressure; SBP, systolic blood pressure. ${ }^{*} P<0.05$.

Table 2 Echocardiographic parameters of the two study groups

\begin{tabular}{lccc}
\hline & Group A $(\mathrm{n}=72)$ & Group $B(\mathrm{n}=46)$ & P-value \\
\hline IVS (mm) & $11.7 \pm 1$ & $11.4 \pm 1.3$ & 0.215 \\
PW (mm) & $11.5 \pm 1$ & $11.3 \pm 1.2$ & 0.390 \\
LAD (mm) & $39.9 \pm 5.7$ & $37.4 \pm 4.2$ & $0.010^{*}$ \\
LVFS (\%) & $28.1 \pm 5.8$ & $31.5 \pm 5.3$ & $0.002^{*}$ \\
LVEF (\%) & $54.1 \pm 8.6$ & $59.2 \pm 7.1$ & $0.001^{*}$ \\
LVMI (g $\left.{ }^{-2}\right)$ & $118.4 \pm 22.2$ & $121.9 \pm 28.3$ & 0.453 \\
RWT & $0.52 \pm 0.058$ & $0.50 \pm 0.088$ & 0.151
\end{tabular}

Abbreviations: IVS, interventricular septal thickness; LAD, left atrial diameter; LVEF, left ventricular ejection fraction; LVFS, left ventricular fractional shortening; LVMI, left ventricular mass index; PW, posterior wall thickness; RWT, relative wall thickness. ${ }^{*} P<0.05$. 
Most interestingly, with respect to the most load-independent LV diastolic performance indices, including Ema/Ama, $\mathrm{Vp}, \mathrm{E} / \mathrm{Vp}$ and $\mathrm{Vp} /$ IVRT, CAD patients had significantly worse values, reflecting the plausible effects of myocardial ischemia on these parameters (all $P<0.05$ ) (Table 3).

After adjusting for confounders, including age, gender, body mass index, arterial hypertension duration, smoking status, dyslipidemia, LV mass index and EF, univariate and multivariate logistic regression analyses demonstrated that IVRT increases were associated with greater odds of CAD, whereas decreases in $\mathrm{Vp}$ and $\mathrm{Vp} / \mathrm{IVRT}$ were associated with lower odds of CAD. However, Ema values were associated with the presence of $\mathrm{CAD}$ in a univariate analysis, but the effect was attenuated in the multivariate analysis (Table 4).

In addition, we estimated the areas under the curve of each echocardiographic index as a crude estimation of the discriminative ability between $\mathrm{CAD}$ and non-CAD patients (Figure 1). As shown in Figure 1, IVRT, Vp, Em and Vp/IVRT strongly discriminated CAD patients from non-CAD patients, as indicated by the reported areas under the curve.

\section{DISCUSSION}

The main finding of this study was that severely depressed LV diastolic function, as suggested by various echocardiographic indices, particularly the $\mathrm{E} / \mathrm{Vp}$ and $\mathrm{Vp} / \mathrm{IVRT}$ ratios, is indicative of $\mathrm{CAD}$ coexistence in patients with uncomplicated essential hypertension.

Arterial hypertension is one of the major risk factors associated with the development of $\mathrm{CAD}$, acting primarily by promoting atherosclerosis. Additionally, it is an important determinant of LV

Table 3 Doppler echocardiographic left ventricular diastolic function indices of the two study groups

\begin{tabular}{|c|c|c|c|}
\hline & Group A $(\mathrm{n}=72)$ & Group $B(\mathrm{n}=46)$ & P-value \\
\hline $\operatorname{Ema}\left(\mathrm{cm} \mathrm{s}^{-1}\right)$ & $8.06 \pm 1.85$ & $7.57 \pm 1.93$ & 0.176 \\
\hline Ema/Ama & $0.66 \pm \pm 0.16$ & $0.75 \pm 0.22$ & 0.014 * \\
\hline $\mathrm{Vp}\left(\mathrm{cm} \mathrm{s}^{-1}\right)$ & $44.2 \pm 15.8$ & $59.7 \pm 20.8$ & $<0.001^{\dagger}$ \\
\hline $\mathrm{E} / \mathrm{Np}$ & $1.28 \pm 0.49$ & $1.10 \pm 0.47$ & $<0.001^{\dagger}$ \\
\hline Vp/IVRT & $0.35 \pm 0.15$ & $0.55 \pm 0.22$ & $<0.001^{\dagger}$ \\
\hline E/Ema & $6.73 \pm 2.26$ & $8.11 \pm 2.25$ & $0.002 *$ \\
\hline IVRT (ms) & $129.2 \pm 13.2$ & $110.5 \pm 14.5$ & $<0.001^{\dagger}$ \\
\hline DT (ms) & $251.9 \pm 59.1$ & $225.5 \pm 57.2$ & 0.019* \\
\hline$E\left(\mathrm{~ms}^{-1}\right)$ & $51.9 \pm 15.3$ & $58.9 \pm 14.2$ & $0.015^{*}$ \\
\hline$A\left(\mathrm{~ms}^{-1}\right)$ & $73 \pm 20$ & $73.2 \pm 18.5$ & 0.962 \\
\hline$E / A$ & $0.74 \pm 0.27$ & $0.83 \pm 0.20$ & 0.068 \\
\hline
\end{tabular}

Abbreviations: A, diastolic mitral inflow wave corresponding to atrial systole; DT, mitral $E$ wave deceleration time; E, early diastolic mitral inflow wave; Ema, TDI-derived lateral mitral annular $\mathrm{E}$ wave; IVRT, isovolumic relaxation time; Vp, flow propagation velocity. ${ }^{*} P<0.05$

$P<0.001$ diastolic dysfunction, and it can be present even before the appearance of LV hypertrophy. ${ }^{1}$ Notably, hypertension-induced myocardial ischemia may have a crucial role in the development of LV diastolic dysfunction even in the case of hemodynamically non-significant coronary stenosis. ${ }^{11}$ In hypertensive subjects, several studies ${ }^{11,12}$ have shown a significant reduction of the coronary flow reserve even in the presence of intact coronary blood flow at rest.

In addition, coronary blood flow is positively related to IVRT, ${ }^{13}$ and intact SERCA function, which facilitates the efflux of calcium ions from the cytoplasm during diastole, is energy-dependent on ATP. Thus, myocardial ischemia may lead to LV relaxation impairment. ${ }^{2}$ In this study, hypertensive patients with CAD exhibited prolonged IVRT as well as lower E mitral inflow and $\mathrm{Vp}$ values compared to patients without $\mathrm{CAD}$, indicating that the intraventricular pressure gradient, which is responsible for normal LV filling, is reduced in the setting of ischemia. ${ }^{14}$

Despite the important contribution of invasive hemodynamic studies in the estimation of LV relaxation, echocardiographic examination prevails in daily clinical practice. Several methods are available, including conventional pulsed Doppler of mitral inflow and pulmonary venous flow, which are highly dependent on LV loading conditions. Consequently, the estimation of LV diastolic performance based solely on these is more or less inadequate and most likely inconclusive. ${ }^{15,16}$ Novel echocardiographic techniques, such as mitral flow propagation velocity and tissue Doppler imaging, are primarily independent of LV preload changes and are considered superior to conventional pulsed Doppler mitral inflow measurements for the comprehensive evaluation of LV diastolic function. ${ }^{15,17,18}$

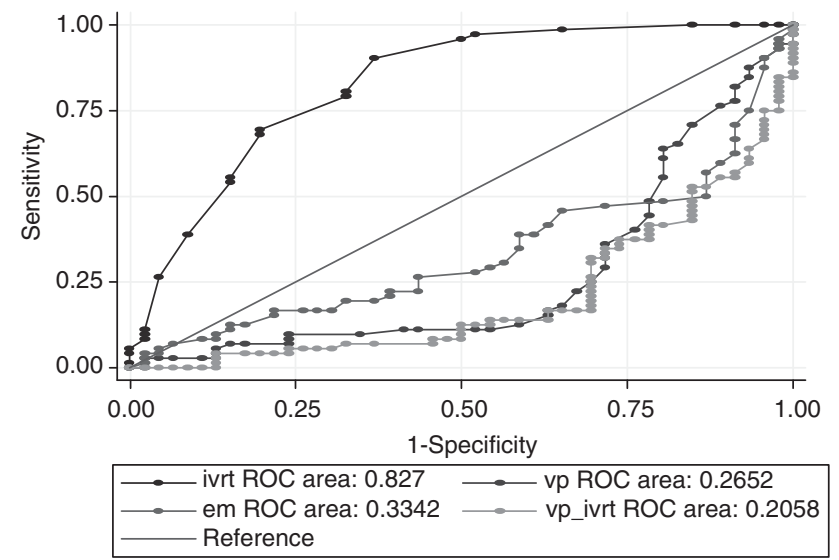

Figure 1 Receiver operating characteristic curves of the IVRT (ivrt), Vp (vp), $\mathrm{Em}$ (em) and Vp/IVRT ( $v p_{-} i v r t$ ) for the discrimination between coronary artery disease (CAD) and non-CAD group patients. A full color version of this figure is available at the Hypertension Research journal online.

Table 4 Results of univariate and multivariate logistic regression a analysis showing the association between diastolic function indices and the presence or not of coronary artery disease

\begin{tabular}{|c|c|c|c|c|c|c|}
\hline Parameter & Crude OR & $95 \% \mathrm{Cl}$ & $\mathrm{P}$-value & Adjusted $^{a} O R$ & $95 \% \mathrm{Cl}$ & $\mathrm{P}$-value \\
\hline IVRT (ms) & 1.102 & $1.062-1.143$ & $<0.001^{\dagger}$ & 1.117 & $1.068-1.168$ & $<0.001^{\dagger}$ \\
\hline $\mathrm{Vp}\left(\mathrm{cm} \mathrm{s}^{-1}\right)$ & 0.954 & $0.931-0.977$ & $<0.001^{\dagger}$ & 0.956 & $0.931-0.982$ & $0.001^{*}$ \\
\hline Vp/IVRT & 0.002 & $0.0001-0.0299$ & $<0.001^{\dagger}$ & 0.002 & $0.0001-0.0372$ & $<0.001^{\dagger}$ \\
\hline Ema $\left(\mathrm{cm} \mathrm{s}^{-1}\right)$ & 0.969 & 0.945-0.994 & $0.018^{*}$ & 0.973 & 0.946-1.002 & 0.073 \\
\hline
\end{tabular}

Abbreviations: $\mathrm{Cl}$, confidence interval; Ema, TDI-derived lateral mitral annular E wave; IVRT, isovolumic relaxation time; OR, odds ratio; Vp, flow propagation.

$* P<0.05$.

${ }^{\dagger} P<0.001$.

${ }^{a}$ Adjusted for age, gender, body mass index, arterial hypertension duration, smoking status, dyslipidemia, LVMI and EF. 
In a study that examined the alteration of LV diastolic function during angioplasty-induced ischemia, an analysis of the flow propagation velocity showed that early filling is highly dependent on the LV relaxation rate, particularly through the phenomenon of asynchrony. ${ }^{19}$ Moreover, in uremic patients, it has been found that a post-dialytic E/FPV ratio $\geqslant 1.5$ predicts a greater risk of adverse cardiovascular events. ${ }^{20}$ However, there is a relative lack of such studies in the setting of essential hypertension.

As hypertension represents a major risk factor for CAD, it is crucial to thoroughly investigate hypertensive patients for the possible presence of myocardial ischemia, as ischemic symptoms in hypertensives may be atypical in nature. Moreover, the standard treadmill exercise test may be inconclusive, particularly in hypertensives with LV hypertrophy, and for this reason, alternative methods such as the echocardiographic stress test are proposed for the detection of ischemia. ${ }^{6}$ This study indicates that impaired LV diastolic function approximated by combined echocardiographic indices, such as the $\mathrm{E} / \mathrm{Vp}$ and $\mathrm{Vp} / \mathrm{IVRT}$ ratios, may reveal the presence of hemodynamically significant coronary stenosis in essential hypertension. A potential clinical implication of this finding is that the abovementioned parameters can be routinely estimated in the echocardiographic examination of high-risk hypertensive patients, strengthening the role of echocardiography beyond the early recognition of LV hypertrophy only.

Our findings may not be globally applicable due to the potential limitations of the present study. The study population was small and selected using the strict inclusion criteria applied by us. Moreover, because there were no data regarding the antihypertensive therapy between the two groups, one could not exclude the possible effects of this treatment on LV diastolic function parameters. Additionally, we have no data regarding the association of echocardiographic indices of diastolic function or the severity of CAD. Further studies are required to determine whether the severity of CAD itself could have an impact on diastolic dysfunction.

In our study, we did not expect to find a lower $\mathrm{E} / \mathrm{E}^{\prime}$ ratio in the $\mathrm{CAD}$ group compared to the non-CAD group. However, there is conflicting evidence regarding the clinical value of this ratio as a marker of diastolic dysfunction. In a recent study, researchers concluded that the suboptimal sensitivity and specificity of the mitral $\mathrm{E} / \mathrm{E}$ ' for predicting increased LV diastolic pressure suggested that the mitral E/E' ratio is of limited clinical value in patients without heart failure. $^{21}$

In conclusion, in the setting of uncomplicated essential hypertension, the early recognition of LV diastolic performance alteration may signal the presence of $\mathrm{CAD}$, indicating the need for a more aggressive approach in terms of pharmacological treatment and invasive evaluation.

\section{CONFLICT OF INTEREST}

The authors declare no conflict of interest.

1 Aeschbacher BC, Hutter D, Fuhrer J, Weidmann P, Delacrétaz E, Allemann Y. Diastolic dysfunction precedes myocardial hypertrophy in the development of hypertension. Am J Hypertens 2001; 14: 106-113.

2 Sasaki O, Hamada M, Hiwada K. Effects of coronary blood flow on left ventricular function in essential hypertensive patients. Hypertens Res 2000; 23: 239-245.
3 Tsang TS, Barnes ME, Gersh BJ, Bailey KR, Seward JB. Risks for atrial fibrillation and congestive heart failure in patients $\geqslant 65$ years of age with abnormal left ventricular diastolic relaxation. Am J Cardiol 2004; 93: 54-58.

4 Aurigemma GP, Gottdiener JS, Shemanski L, Gardin J, Kitzman D. Predictive value of systolic and diastolic function for incident heart failure in the elderly: the Cardiovascular Health Study. J Am Coll Cardiol 2001; 37: 1042-1048.

5 Redfield MM, Jacobsen SJ, Burnett JC Jr, Mahoney DW, Bailey KR, Rodeheffer RJ. Burden of systolic and diastolic ventricular dysfunction in the community: appreciating the scope of the heart failure epidemic. JAMA 2003; 289: 194-202.

6 Mancia G, De Backer G, Dominiczak A, Cifkova R, Fagard R, Germano G, Grassi G, Heagerty AM, Kjeldsen SE, Laurent S, Narkiewicz K, Ruilope L, Rynkiewicz A Schmieder RE, Struijker Boudier HA, Zanchetti A, Vahanian A, Camm J, De Caterina R, Dean V, Dickstein K, Filippatos G, Funck-Brentano C, Hellemans I, Kristensen SD, McGregor K, Sechtem U, Silber S, Tendera M, Widimsky P, Zamorano JL, Kjeldsen SE, Erdine S, Narkiewicz K, Kiowski W, Agabiti-Rosei E, Ambrosioni E, Cifkova R, Dominiczak A, Fagard R, Heagerty AM, Laurent S, Lindholm LH, Mancia G, Manolis A, Nilsson PM, Redon J, Schmieder RE, Struijker-Boudier HA, Viigimaa M, Filippatos G, Adamopoulos S, Agabiti-Rosei E, Ambrosioni E, Bertomeu V, Clement D, Erdine S, Farsang C, Gaita D, Kiowski W, Lip G, Mallion JM, Manolis AJ, Nilsson PM, O'Brien E, Ponikowski P, Redon J, Ruschitzka F, Tamargo J, van Zwieten P, Viigimaa M, Waeber B, Williams B, Zamorano JL. 2007 Guidelines for the management of arterial hypertension: The Task Force for the Management of Arterial Hypertension of the European Society of Hypertension (ESH) and of the European Society of Cardiology (ESC). Eur Heart J 2007; 28: 1462-1536.

7 Farias CA, Rodriguez L, Garcia MJ, Sun JP, Klein AL, Thomas JD. Assessment of diastolic function by tissue Doppler echocardiography: comparison with standard transmitral and pulmonary venous flow. J Am Soc Echocardiogr 1999; 12: 609-617.

8 De Boeck WL, Cramer MJ, Oh JK. Spectral pulsed tissue Doppler imaging in diastole: a tool to increase our insight in and assessment of diastolic relaxation of the left ventricle. Am Heart J 2003; 146: 411-419.

9 Lang RM, Bierig M, Devereux RB, Flachskampf FA, Foster E, Pellikka PA, Picard MH, Roman MJ, Seward J, Shanewise JS, Solomon SD, Spencer KT, Sutton MS, Stewart WJ. Recommendations for chamber quantification: a report from the American Society of Echocardiography's Guidelines and Standards Committee and the Chamber Quantification Writing Group, developed in conjunction with the European Association of Echocardiography, a branch of the European Society of Cardiology. J Am Soc Echocardiogr 2005; 18: 1440-1463.

10 Devereux RB, Reichek N. Echocardiographic determination of left ventricular mass in man. Anatomic validation of the method. Circulation 1977; 55: 613-618.

11 Hamouda MS, Kassem HK, Salama M, El Masry M, Shaaban N, Sadek E, Khandheria BK, Seward JB, Elhendy A. Evaluation of coronary flow reserve in hypertensive patients by dipyridamole transesophageal Doppler echocardiography. Am J Cardiol 2000; 86 305-308.

12 Palombo C, Kozakova M, Magagna A, Bigalli G, Morizzo C, Ghiadoni L, Virdis A, Emdin M, Taddei S, L'Abbate A, Salvetti A. Early impairment of coronary flow reserve and increase in minimum coronary resistance in borderline hypertension. I Hypertens 2000; 18: 453-459.

13 Frohlich ED. Risk mechanisms in hypertensive heart disease. Hypertension 1999; 34 782-789

14 Lester SJ, Tajik AJ, Nishimura RA, Oh JK, Khandheria BK, Seward JB. Unlocking the mysteries of diastolic dysfunction: deciphering the Rosetta Stone 10 years later. J Am Coll Cardiol 2008; 51: 679-689.

15 Sohn DW, Chai IH, Lee DJ, Kim HC, Kim HS, Oh BH, Lee MM, Park YB, Choi YS Seo JD, Lee YW. Assessment of mitral annulus velocity by Doppler tissue imaging in the evaluation of left ventricular diastolic function. J Am Coll Cardiol 1997; 30: 474-480.

16 Nagueh SF, Middleton KJ, Kopelen HA, Zoghbi WA, Quiñones MA. Doppler tissue imaging: a technique for evaluation of left ventricular relaxation and estimation of filling pressures. J Am Coll Cardiol 1997; 30: 1527-1533.

17 Garcia M, Thomas JD, Klein AL. New Doppler echocardiographic applications for the study of diastolic function. J Am Coll Cardiol 1998; 32: 865-875.

18 Farias CA, Rodriguez L, Garcia MJ, Sun JP, Klein AL, Thomas JD. Assesment of diastolic function by tissue Doppler echocardiography: comparison with standard transmitral and pulmonary venous flow. J Am Soc Echocardiogr 1999; 12 609-617.

19 Duval-Moulin AM, Dupouy P, Brun P, Zhuang F, Pelle G, Perez Y, Teiger E, Castaigne A, Gueret $\mathrm{P}$, Dubois-Randé JL. Alteration of left ventricular diastolic function during coronary angioplasty-induced ischemia: a color M-mode Doppler study. J Am Coll Cardiol 1997; 29: 1246-1255.

20 Hsiao SH, Huang WC, Chiou KR, Lee CY, Yang SH, Wang WC, Lin SK. Major events in uremic patients: insight from parameters derived by flow propagation velocity. J Am Soc Echocardiogr 2008; 21: 741-746.

21 Previtali M, Chieffo E, Ferrario M, Klersy C. Is mitral E/E' ratio a reliable predictor of left ventricular diastolic pressures in patients without heart failure? Eur Heart Cardiovasc Imaging 2012; 13: 588-595. 